\section{Hyoid Arch of Rhinobatidæ}

THE skeleton of the hyoid arch of Batoidei has been for many years the subject of discussion by morphologists. Gegenbaur ${ }^{1}$ considered the hyoid elements bearing hyal rays and lying posterior to the hyomandibular cartilage to represent the ceratohyal of Selachoidei, which became disconnected from the ventral edge of the hyomandibular and extended upwards behind it. Krivetski ${ }^{2}$ and de Beer ${ }^{3}$ considered these hyoid elements as pseudohyoid and formed by the fusion of the proximal ends of the hyal rays. Such a phenomenon occurred during the phylogenetic divergence between Batoidei and Selachoidei, and was largely associated with the increased distance between the mouth and first gill slit in Batoidei. Thus the pseudohyoid elements were necessary for providing a support for the hyal rays, as well as the anterior wall of the first gill slit. During the enlargement and extension of these pseudohyoid elements, the original ceratohyal in Batoidei has disappeared.

The study of the hyoid arch of Rhinobatidæ, as represented by Rhinobatus halavi and Rhynchobatus djiddensis from the Red Sea, supports the view of both Krivetski and de Beer. As a matter of fact, both Rhinobatus halavi and Rhynchobatus djiddensis are transitional forms between Selachoidei and Batoidei (Bridge, Regan ${ }^{5}$ and Goodrich ${ }^{6}$ ). It is found that in these two fishes the original ceratohyal, which has completely disappeared in specialized Batoidei, is still persisting in addition to the pseudohyoid. This ceratohyal, which is relatively much reduced, bears the correct relations of a selachoid ceratohyal to the afferent hyoidean artery, being immediately dorsal to it. Also it is still bearing hyal rays. The originally direct connexion between the hyomandibular and ceratohyal of Selachoidei can be traced by the presence of a ligamentous vestige between the hyomandibular and this reduced ceratohyal. Also the afferent pseudobranchial artery, on running forwards from the efferent hyoidean artery, is median to the pseudohyoid, whereas it is lateral to both the hyomandibular cartilage and the reduced ceratohyal.

Thus the pseudohyoid is a structure altogether distinct from the ceratohyal. Accordingly, the peculiar articulation between the ventral pseudohyoid bar and the reduced ceratohyal is a secondary one. It is gained by the depression of the ceratohyal due to the ventral extension of the ventral pseudohyoid bar. That such an extension has taken place is supported by the fact

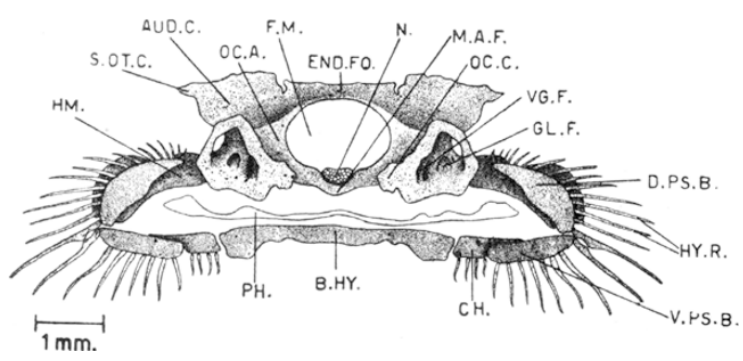

Rhinobatus halavi. Reconstructed posterior view of neurocranium and hyoid arch. $A U D . C .$, auditory capsule ; $B . H Y . .$, basihyal ; $C H .$, ceratohyal ;
$D . P S . B .$, dorsal pseudohyoid bar $E N D . F O .$, endolymphatic fossa ; $F . M$., foramen magnum ; GL.F., glossopharyngeal foramen ; $H M$. hyomandibular cartilage; HY.R., hyal rays $M . A . F$. median articular facet for anterior vertebral plate; $N$. notochord ; OC.A., occipital arch ; OC.C., occipital condyle PH., pharynx; S.OT.C., supraotic crest; V.PS.B., ventra pseudohyoid bar ; VG.F., vagus foramen that the more ventral hyal rays are loosely attached to the ventral pseudohyoid bar while the dorsal rays are completely fused with it. It is to be noted also that the amount of depression of the ceratohyal in Rhinobatus halavi is relatively greater than that in Rhynchobatus djiddensis. This shows that these transitional forms represent different steps towards the highly specialized Batoidei, where the ceratohyal is completely missing. The condition of Rhinobatus halavi, possessing a smaller ceratohyal, seems to be somewhat beyond that of Rhynchobatus djiddensis towards the more specialized Batoidei. This agrees with the conclusion arrived at by Melouk ${ }^{7}$ from embryological considerations.

\section{A. R. HAMDY}

\section{Department of Zoology, \\ Faculty of Science, \\ Fouad I University, Cairo. \\ May 24.}

1 Gegenbaur, C., "Das Kopfskelet der Selachier" (Ieipzig, 1872).

${ }^{2}$ Krivetski, A., Rev. Zool. Russe, 2 (1917).

s de Beer, G. R., Quart. J. Micro. Sci., 75 (19:32).

"Bridge, J. W., "Cambridge Nat. Hist." (Fishes), 7 (London, 1904).

5 Regan, C. T., Proc, Zool. Soc. (London, 1906).

"Goodrich, E. S., "A Treatise on Zoology" (London, 1909).

"Melouk, M. A., Pub. Mar. Biol. Station, Ghardaqa, 7 (1949).

\section{Epidermal Glands in Centipedes}

THE epidermis of a centipede is virtually an epithelium of unicellular glands, their secretion being extruded over the surface of the cuticle ${ }^{1}$. In addition to the general epidermal glands, there are local groups of much larger glands histologically similar to the generally distributed glands. Their secretions are chemically similar and, furthermore, they have similar properties to the sclerotin and pro-sclerotin ${ }^{1}$ of the cuticle.

Opening on the sternites of Geophilomorphs are batteries of large columnar gland cells. The secretion from these sternal glands in Haplophilus subterraneus is a white mobile fluid when extruded, but it rapidly hardens into an amber-coloured material on exposure to the air. Attems ${ }^{2}$ has described a similar secretion from the sternal glands of Himantarium gabrielis. Similar batteries of glands are found in Lithobiomorphs, though they do not appear to have been described. They open by numerous pores on the inner, morphologically posterior face of the four distal podomeres (femur, tibia, tarsus and metatarsus) of the last two pairs of legs. They may be referred to as the 'telopodal glands'. Like that of the sternal glands their secretion hardens on exposure, forming a material which is amber-coloured when seen in mass, but is normally extruded as long sticky fibres resembling silk. This secretion has been noted by Verhoeff $^{3}$ and Willem, although they both state incorrectly that the material is extruded from the coxal glands ('glandes filières' of Willem).

Telopodal glands have been examined in several species of Lithobius and in the related genus Lamyctes. Like the general epidermal gland secretions ${ }^{1}$, that of the sternal and telopodal glands colours with sudan black. It is intensely positive to Millon's reagent and also gives a positive ninhydrin test. The secretion thus appears to be a lipoid-protein complex or mixture. It is very similar in this respect to the sclerotin and pro-sclerotin of the cuticle, in which phenolic groups, probably tyrosine residues, are 